\title{
Auxiliary Aids to Alleviate Pain and Anxiety during Local Anesthesia Administration: A Comparative Study
}

\author{
Vishu Midha ${ }^{1}$, Vasu Midha², Rohini Dua ${ }^{3}$, Ripin Garewal ${ }^{4}$, Anuraj S Kochhar ${ }^{5}$, Gulsheen K Kochhar ${ }^{6}$
}

\begin{abstract}
Introduction: In dentistry, pain and anxiety have been the most challenging aspect in the management of a pediatric patient. When every effort to perform local anesthesia (LA) is not successful, the result would be more stressful for both the dentist and the patient. The so-called clichéd paradigm that "pain and dentistry are inseparable" can be resolved by updating the knowledge and skills of the practitioner by using the more advanced techniques in controlling and managing the pain. An array of techniques for administering the LA to improve the comfort level of our patients has been the area of interest.

Aim and objective: To evaluate and compare the efficacy of various adjunctive aids of LA in reducing pain and anxiety in pediatric patients of the 6-8 years age-group. A total of 90 child dental patients were selected and randomly divided into six groups, i.e., control, topical gel, audio, audiovisual, transcutaneous electric nerve stimulation (TENS), and Vibraject group. Physiological parameters, psychological parameters, and pain assessment were recorded.
\end{abstract}

Results: As reflected by the results, the minimum pulse rate "during" and "after" LA administration is seen in A/V (D) and TENS (E) "during" LA administration. Children were less anxious and more relaxed in the audiovisual group and TENS group. Transcutaneous electric nerve stimulation and Vibraject groups showed maximum reduction in pain.

Conclusion: A/V (D) and TENS (E) groups exhibited the least anxiety. Also, the minimum pain was felt using TENS (E) and Vibraject (F) and hence, may be considered as adjunctive aids in pain reduction during LA administration.

Keywords: Anxiety, Audio aids, Audiovisual aids, Local anesthesia, Pain, Transcutaneous electric nerve stimulation, Topical gel, Vibraject.

International Journal of Clinical Pediatric Dentistry (2021): 10.5005/jp-journals-10005-1935

\section{INTRODUCTION}

Pain has been a constant torturer of mankind for ages. The most common means to restrain pain is the use of local anesthesia (LA) during dental procedures. ${ }^{1}$ Local anesthesia is the linchpin of modern dentistry but the injection of LA is the considerable source of a child's fear and anxiety. The control of pain during dental procedures especially for a child is one of the most important constituents supporting sound principles of behavior management. Numerous advanced techniques for delivery of local anesthetic to control the pain for the child are provided in most instances using skilled and sensible procedures. The phrase "DOUBLE EDGE SWORD" in dentistry is commonly used for PAIN which means that the pain is the main reason for bringing the patient to a dental clinic and on the other hand fear of pain causes the patient for driving him away from the dental care.

Various resources and procedures are used to alleviate the sensation of discomfort produced by needle insertion such as distraction aids. Anxiety and fear of pain and the actual sensation of pain have a strong correlation. ${ }^{2}$ A wide array reducing pain, which includes audiovisual, i.e., from video games, and audio distraction, to watching television, cartoons, and stories to most recent virtual reality distraction aids. ${ }^{3-7}$ Audiovisual distraction (AVD)-virtual reality 3D Box completely obstructs the dental environment and does not interfere with dental treatment. Another group of resources is the prior surface application of topical anesthetic on oral mucosa by the use of topical gel. Topical anesthetics are available in different forms such as ointment, gel, liquid, and pressurized spray forms. In addition, mechanical resources are also used such as generating sensations to distract the child's attention and temporarily blocking the
${ }^{1}$ Department of Pediatric Dentistry, National Dental College and Hospital, Dera Bassi, Punjab, India

${ }^{2}$ Department of Conservative Dentistry and Endodontics, National Dental College and Hospital, Dera Bassi, Punjab, India

${ }^{3,6}$ Department of Pedodontic and Preventive Dentistry, National Dental College and Hospital, Dera Bassi, Punjab, India

${ }^{4}$ Department of Pediatric and Preventive Dentistry, National Dental College and Hospital, Dera Bassi, Punjab, India

${ }^{5}$ DSATP Student in Orthodontics and Dentofacial Orthopedics, Faculty of Dentistry, University of Toronto, Canada

Corresponding Author: Gulsheen K Kochhar, Department of Pedodontics and Preventive Dentistry, National Dental College and Hospital, Dera Bassi, Punjab, India, Phone: +91 9888416164, e-mail: gulsheenuppal@gmail.com

How to cite this article: Midha V, Midha V, Dua R, et al. Auxiliary Aids to Alleviate Pain and Anxiety during Local Anesthesia Administration: A Comparative Study. Int J Clin Pediatr Dent 2021;14(1):104-108.

Source of support: Nil

Conflict of interest: None

transmission of nociceptive messages such as vibration and pressure [e.g., transcutaneous electric nerve stimulation (TENS) and vibrotactile devices]. Electronic dental anesthesia/TENS is a therapy that uses a mild amount of electric current to produce dental anesthesia. Some of the recent advances of local anesthetic delivery systems through the use of vibration thus reducing pain called vibrotactile devices which is aimed at reducing the fear of the needle. This device is based on theory "gate control theory of pain management" Melzac and Wall ${ }^{8}$ stating that pain can be 
decreased by concurrently activation of nerve fibers through vibration. Some of the vibrotactile devices are vibraject, dental vibe, and accupal. Vibraject LLC (USA) was first patented by an oral surgeon Dr Norman Pokran in 1995. Vibraject is an attachment to the dental injection which has vibration and works on gatecontrol theory.

Unfortunately, the technique used to control dental procedure pain itself produces distress. So, for all the practitioners, the aim should be to reduce injection sensation to a minimum to alleviate the apprehension and fear from the patient's mind. ${ }^{9}$ With the increasing incidence of dental problems, there is an increased requirement for $L A$. Reducing the pain with various adjunctive aids will be beneficial for alleviating pain and anxiety in pediatric patients..$^{10}$ The knowledge of the latest upcoming methods of LA delivery systems for the pediatric dentist is of utmost importance that will definitely help them manage a painless dental practice and help inculcate a positive lookout for dental procedures among patients. ${ }^{11}$

The various complementary methods which were used in the study - topical gel, audio aids, audiovisual aids, TENS, and vibratory device. The aim of the study was to evaluate and compare the efficacy of various adjunctive aids of LA in reducing pain and anxiety in pediatric dental patients.

\section{Materials and Methods}

A total of 90 children were included in the study of the $6-8$ years of age group, with 15 children in each subgroup, were selected from the outpatient Department of Paediatric and Preventive Dentistry, National Dental College and Hospital, Dera Bassi, Punjab. The outline of the study was approved by the ethical committee of the National Dental College and Hospital, Dera Bassi.
The study aimed to evaluate and compare the efficacy of various adjunctive aids of LA in reducing pain and anxiety in pediatric dental patients.

Patients were screened according to pre-decided inclusion and exclusion criteria. Adjunctive aids used were topical gel, audio aids, audiovisual aids, TENS, and Vibraject.

\section{Inclusion Criteria}

- Children between the age group of 6 years and 8 years were selected.

- Patients requiring LA for dental procedures such as deep filling, pulpotomy, pulpectomy, or extraction procedure.

- Potentially cooperative patients.

\section{Exclusion Criteria}

- Medically and physically compromised children.

- Exclusion criteria for TENS group patients

- Pacemakers.

- Allergic response to the electrode/tape/gel.

- Skin conditions (e.g., eczema, dermatitis).

- Current or recent bleeding/hemorrhage.

- Open wounds.

- Compromised circulation, e.g., ischemic tissue, thrombosis, and associated conditions.

Based on the above-mentioned criteria, a total of 90 patients were finally selected for the study and the subjects were divided into six different groups-in group I control group, in group II topical gel, in group III audio aids, in group IV audiovisual aids, in group V TENS, and in group VI Vibraject was used (Fig. 1). Samples were divided into six groups.

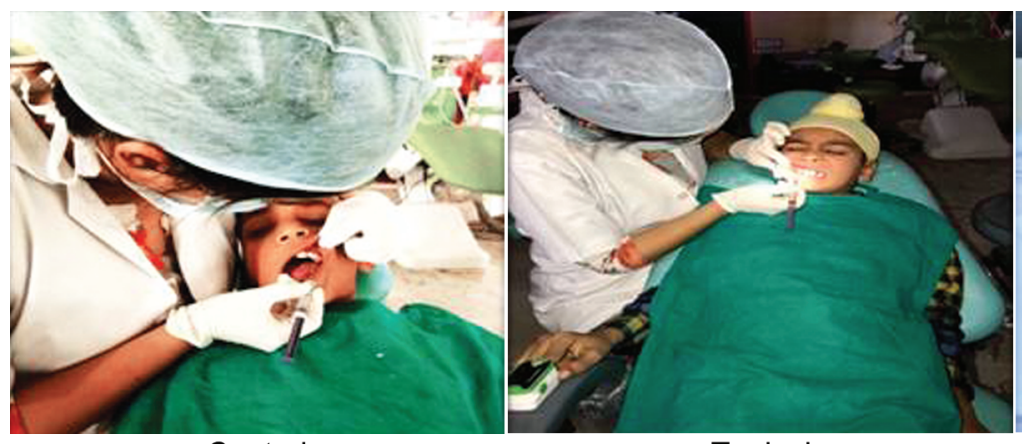

Control

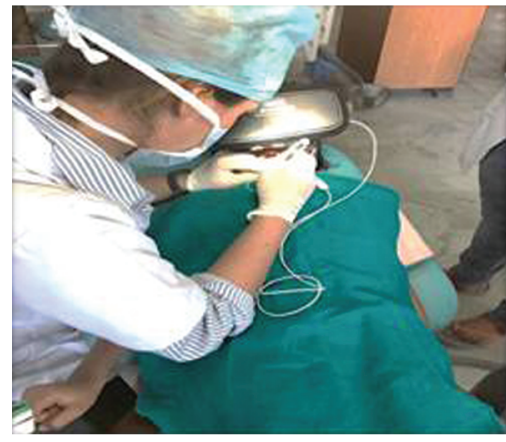

Audiovisual
Topical

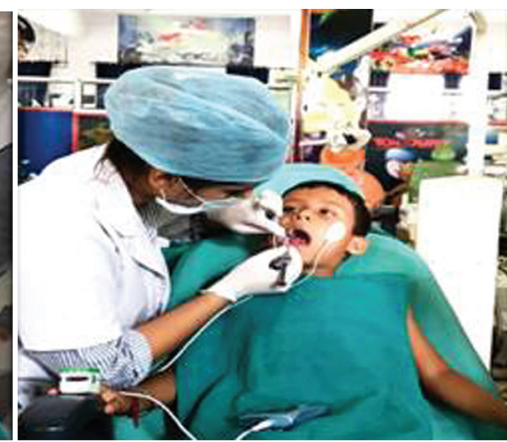

Tens

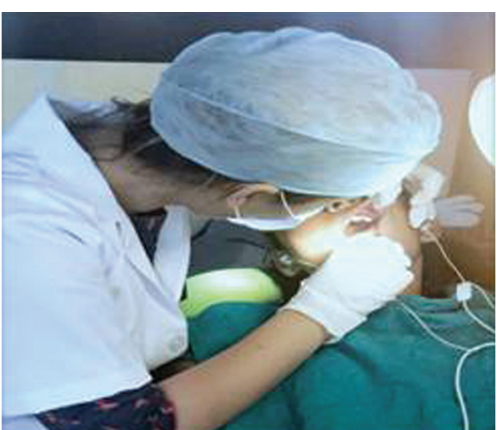

Audio

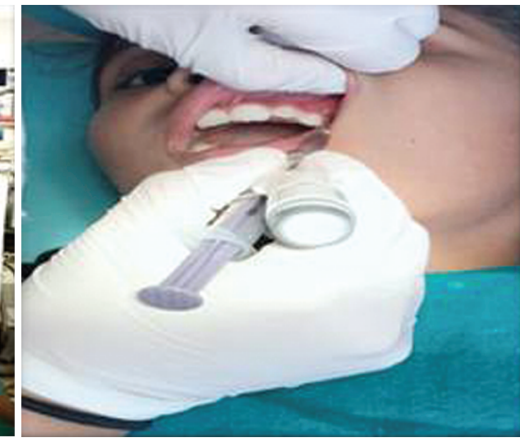

Vibraject

Fig. 1: Samples divided into six groups 
Before enrolment in the study, proper informed consent was taken from the parents of all participants.

- The patient's oral cavity was examined requiring LA for dental procedures such as deep filling, pulpotomy, pulpectomy, or extraction procedure with the help of a mouth mirror and explorer/probe. Before administration of LA, pre-records were taken for physiological, psychological, and behavioral parameters. During the administration of LA, physiological parameter was measured using pulse oximeter. After LA was administered, readings for the physiological, psychological, behavioral, and pain rating scale were recorded.

- A combination of the following anxiety measuring parameters was used to assess the child anxiety level in each visit:

- Physiological parameters

- Pulse rate.

- Psychological parameters

- Fear assessment-Dental Sub-scale of Children's Fear Survey Schedule-Short Scale (DFSS-SF).

- Behavior assessment-Modified Child Dental Anxiety scale (MCDAS).

- Pain assessment scales-Wong-Baker FACES Pain Rating Scale (WBFS).

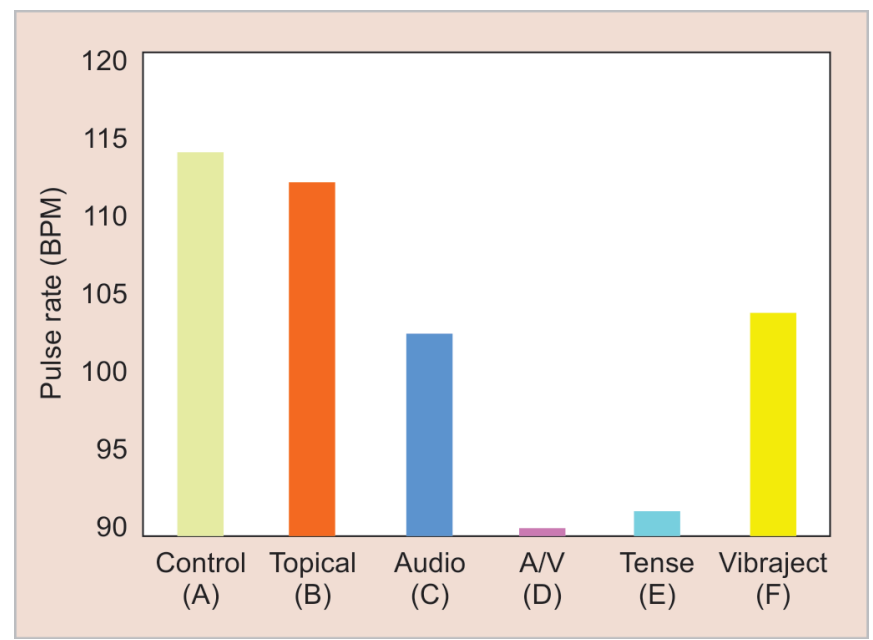

Fig. 3: Mean pulse rate among different subgroups "after" LA administration

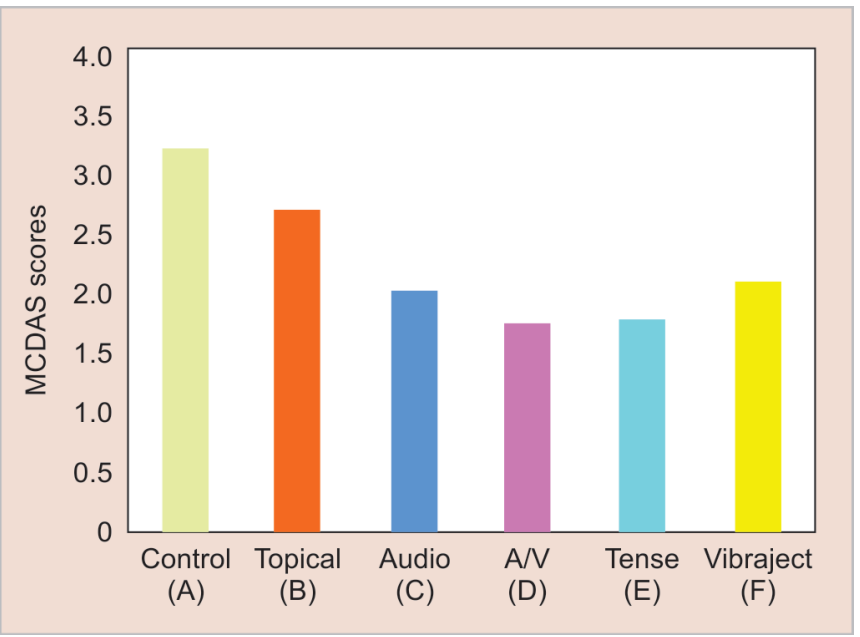

Fig. 5: Mean scores for modified child dental anxiety scale (MCDAS) "after" LA administration

\section{Results (Figs 2 to 6).}

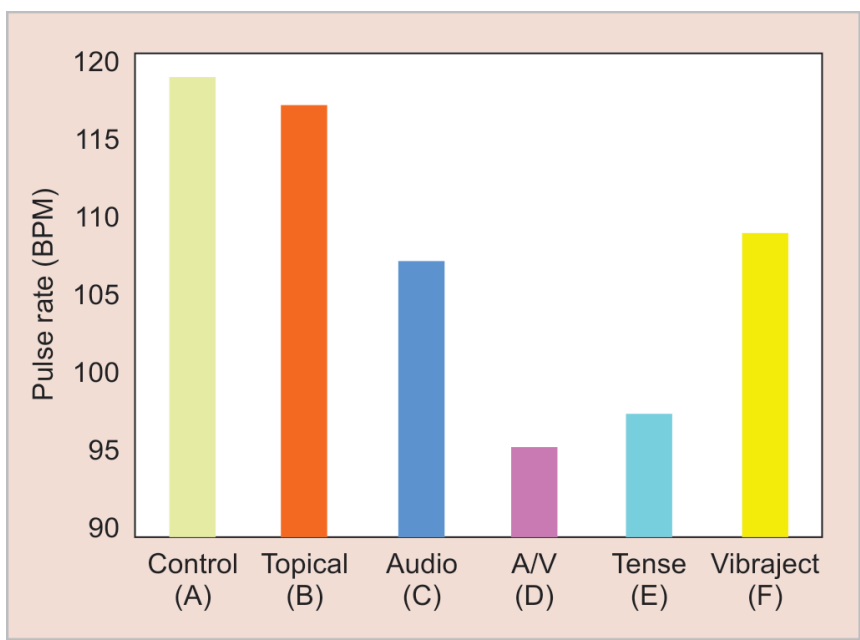

Fig. 2: Mean scores for pulse rate among different subgroups "during" LA administration

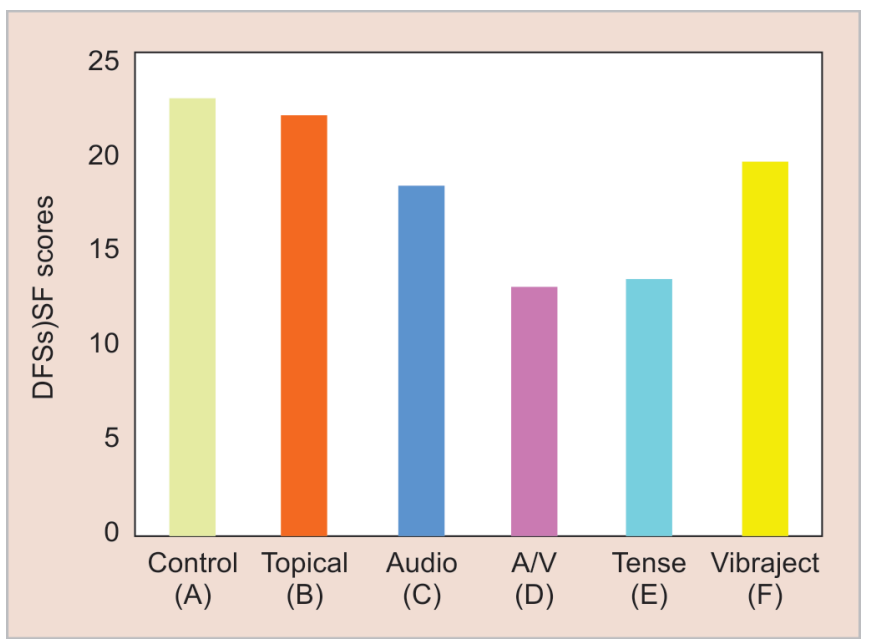

Fig. 4: Mean scores for dental sub-scale of children fear survey scheduleshort scale (DFSS-SF) "after" LA administration

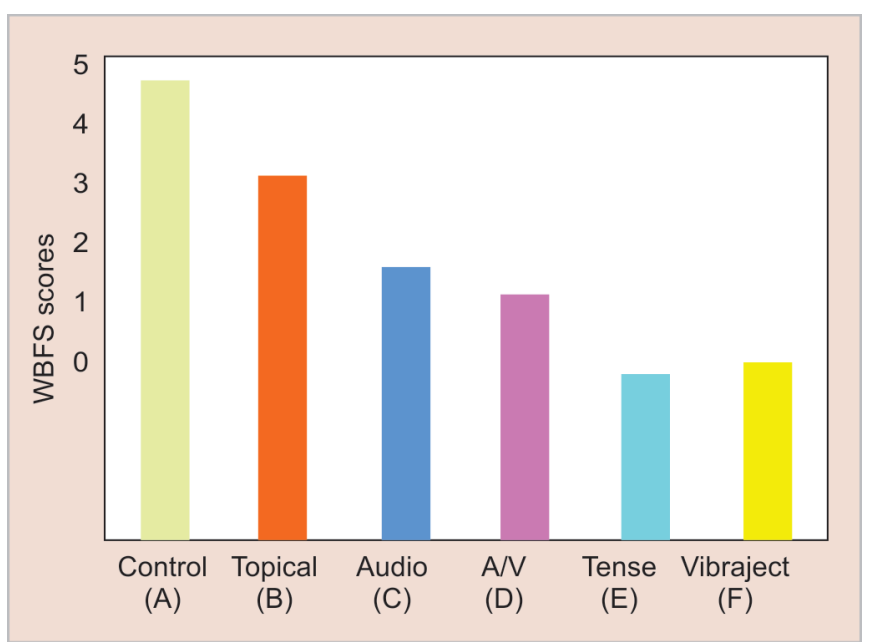

Fig. 6: Mean scores for Wong-Baker FACES pain rating scale (WBFS) "after" LA administration 


\section{Discussion}

Although LA is the "backbone" of a modern dental practice, perhaps the use of local anesthetic injection is the significant source of patient's fear and anxiety. ${ }^{12}$ Similarly, a study by Angelo and Polyvios stated that the pain and anxiety are interconnected, i.e., most pain control is achieved typically by the administration of $L A$, which is the highly effective method, and on the flip side it is all the most anxiety-provoking procedure for all the patients. ${ }^{13}$

To obtain adequate pain control with miniscule discomfort has been a significant concern for dentists. The foremost skill required for all dental surgeons is the ability to provide an efficacious and most importantly pain-free LA. ${ }^{14}$

When the mean pulse rate recorded "Before", the LA for all six groups of 6-8 years of age were compared no statistically significant difference was observed. Thus, indicating that all the groups had comparable pulse rate and anxiety level "Before" the LA administration. The mean pulse rate recorded "During" and "After" the LA administration for all the six groups when compared (Figs 2 and 3 ) revealed a statistically significant difference. The results revealed that pulse rate in Control (A) and Topical (B) was significantly higher as compared to other groups followed by Audio (C) and Vibraject (F) which exhibited non-significant differences. The minimum pulse rate was seen in A/V (D) and TENS (E) "during" LA administration. So, the children were more relaxed in the audiovisual group and TENS group followed by Vibraject and audio group and were least relaxed in topical and control group "During" and "After" the LA administration.

Similar results were found in Prabhakar et al. ${ }^{15}$ study which showed statistically significant differences between their subgroups, i.e., control group, audio, and audio-visual group.

The significant difference in pulse rate values between the control group and audio group was not in accordance with Aitken et al.'s ${ }^{16}$ results in which no significant difference in pulse rate between the control group, upbeat music group, and relaxing music group.

When the mean DFSS-SF recorded "Before", the LA administration for all the six groups statistically insignificant difference was observed. When the mean DFSS-SF recorded "After", the LA administration was compared (Fig. 4), a statistically significant difference was observed. The results indicated that DFSS-SF in Control (A) and Topical (B) was significantly higher as compared to other groups. However, A/V (D), TENS (E), Audio (C), and Vibraject (F) exhibited the least DFSS scores. It indicated that children were more relaxed in the audio-visual group and TENS group as compared to the audio group and Vibraject group followed by topical gel and control group "After" the LA administration. This study concluded that anxiety was least while using 3D virtual eyeglasses during LA administration the children gained control over the unpleasant stimulus and a familiar environment was induced through 3D eyeglasses. ${ }^{17}$ Also, multi-sensory distraction was achieved as the child concentrate on the video, thereby distracting out of dental treatment, and the unpleasant dental sounds were eliminated such as the sound of air rotor or suction with the help of ongoing program sounds. 4,18

When the mean MCDAS has recorded "Before", the LA administration for all the six groups statistically insignificant difference was observed. When the mean MCDAS scores "After", the LA administration was compared (Fig. 5), a statistically significant difference was observed. The results indicated revealed that MCDAS in Control (A) and Topical (B) was significantly higher as compared to other groups. However, A/V (D), TENS (E), Audio (C), and Vibraject (F) exhibited the least DFSS scores. It indicated that children were more relaxed in the audio-visual group and TENS group followed by audio group and Vibraject group and most anxious in topical gel and control group "After" the LA administration. It was in accordance with the study done by Ram et al. ${ }^{19}$ who also found that 3D virtual eyeglasses were a successful distraction aid for the easement of the undesirable and discomfort that arises during procedures.

When the mean WBFS recorded "After", the LA administration were compared statistically significant difference was observed as (Fig. 6). The results revealed that WBFS in Control (A) and Topical (B) was significantly higher followed by the Audio (C) group. The minimum WBFS scores were seen in TENS (E), Vibraject (F), AND A/V (D) "after" LA administration. Cho et al. ${ }^{20}$ and Lodaya ${ }^{21}$ suggested TENS be a useful supplement in imparting painless anesthesia during pediatric dental procedures. Also, studies on the effectiveness of vibratory devices have been done and concluded that during LA injection, vibration is regarded as a practical technique that helps in the reduction of pain and anxiety. ${ }^{22}$ Our result was not in accordance with studies conducted by Dr Bhawana which concluded that no statistically significant difference in pain intensity between different techniques, i.e., Vibraject and conventional syringe during LA deposition. ${ }^{23}$

\section{Conclusion}

- So, the present study reported that the pulse rate "during" and "after" LA administration minimum pulse rate was seen in A/V (D) and TENS (E) "during" LA administration. Hence, children were most relaxed in the audio-visual group and TENS group followed by Vibraject and audio group, and least relaxed in the topical gel and control group "during" and "after" the LA administration. Thus, audiovisual aids and TENS proved to be effective adjuncts of LA administration in anxious pediatric patients.

- Children were less anxious and fearful and more relaxed as depicted by DFSS-SF and MCDAS in the audio-visual group and TENS group followed by audio group and Vibraject group and most anxious in topical gel and control group "After" the LA administration. Thus, audio-visual aids and TENS group were found to be more effective in the reduction of anxiety as compared to other adjunctive aids of $L A$ administration.

- The results revealed that WBFS in Control (A) and Topical (B) was significantly higher followed by the Audio (C) group. The minimum WBFS scores were seen in TENS (E), Vibraject (F), and A/V (D). Thus, the TENS and Vibraject group showed the maximum reduction in pain, and the maximum pain was felt in the topical gel and control group. Therefore, it is suggested TENS and Vibraject may be considered effective adjunctive aids in pain reduction during $L A$ administration.

\section{References}

1. Kasat V, Gupta A, Ladda R, et al. Transcutaneous electric nerve stimulation (TENS) in dentistry- a review. J Clin Experiment Dentis 2014;6(5):e562-e568. DOI: 10.4317/jced.51586.

2. Oliveira ACA, Amorim KS, Nascimento Júnior EM, et al. Assessment of anesthetic properties and pain during needleless jet injection 
anesthesia: a randomized clinical trial. J Appl Oral Sci [online] 2019;27:e20180195. DOI: 10.1590/1678-7757-2018-0195.

3. Corah NL, Gale EN, Illig SJ. Psychological stress reduction dental procedures. J Dent Res 1979;5(4):1347-1351. DOI: 10.1177/00220345790580040801.

4. Seyrek SK, Corah NL, Pace LF. Comparison of three distraction techniques in reducing stress in dental patients. J Am Dent Assoc 1984;108(3):327-329. DOI: 10.14219/jada.archive.1984.0034.

5. Carlin S, Ward WD, Gershon A, et al. Sound stimulation and its effect on dental sensation threshold. Science 1962;138(3546):1258-1259. DOI: $10.1126 /$ science.138.3546.1258.

6. Weisenberg M, Tepper I, Schwarzwald J. Humor as a cognitive technique for increasing pain tolerance. Pain 1995;63(2):207-212. DOI: 10.1016/0304-3959(95)00046-U.

7. Stark LJ, Allen KD, Hurst $M$, et al. Distraction: its utilization and efficacy with children undergoing dental treatment. J Appl Behav Anal 1989;22(3):297-307. DOI: 10.1901/jaba.1989. 22-297.

8. Melzack R, Wall PD. Pain mechanisms: a new theory. Science 1965;150(3699):971-979. DOI: 10.1126/science.150.3699.971.

9. Singh N, Agarwal S, Bhagchandani J, et al. Painless anesthesia: a new approach. J Dentofac Sci 2013;2(2):49-55.

10. Peedikayil FC, Vijayan A. An update on local anesthesia for pediatric dental patients. Anesthe Essays Res 2013;7(1):4-9. DOI: 10.4103/02591162.113977.

11. Kulkarni N. Painless anaesthesia in pediatric dentistry: an updated review. J Dent Med Sci (IOSR-JDMS) 2019;18(4):67-71.

12. Weinstein P, Milgrom P, Getz T. Treating fearful dental patients: a practical behavioral approach. J Dent Pract Adm 1987;4(4):140-147.

13. Angelo $Z$, Polyvios $C$. Alternative practices of achieving anaesthesia for dental procedures: a review. J Dent Anesth Pain Med 2018;18(2): 79-88. DOI: 10.17245/jdapm.2018.18.2.79.
14. Kaufman $E$, Weinstein $P$, Milgrom P. Difficulties in achieving local anesthesia. J Am Dent Associat 1984;108(2):205-208. DOI: 10.14219/ jada.archive.1984.0470.

15. Prabhakar AR, Marwah N, Raju OS. A Comparison between audio and audio-visual distraction techniques in the management of anxious pediatric dental patients. J Indian Soc Pedod Prevent Dentis 2007;25(4):177-182. DOI: 10.4103/0970-4388.37014.

16. Aitken JC, Wilson S, Coury D, et al. The effect of music distraction on pain, anxiety and behavior in pediatric dental patients. Pediatr Dent 2002;24(2):114-118.

17. Klein SA, Winkelstein ML. Enhancing pediatric health care with music. J Pediat Health Care 1996;10(2):74-81. DOI: 10.1016/S08915245(96)90030-9.

18. Baghdadi ZD. Evaluation of audio analgesia for restorative care in children treated using electronic dental anesthesia. J Clin Pediat Dentis 2000;25(1):9-12.

19. Ram D, Shapira J, Holan G, et al. Audiovisual video eyeglass distraction during dental treatment in children. Quintessence Int 2010;41(8):673679.

20. Cho SY, Drummond BK, Anderson MH, et al. Effectiveness of electronic dental anesthesia for restorative care in children. Pediat Dentis 1998;20(2):105-111.

21. Lodaya R, Bhat C, Gugwad SC, et al. Clinical evaluation of transcutaneous electrical nerve stimulation (TENS) for various treatment procedures in pediatric dentistry. Int J Clin Dent Sci 2010;1:20-25.

22. Ungor C, Tosun E, Dayisoylu EH, et al. The effects of vibration on pain and anxiety during local anesthesia administration. JSM Dent 2014;2(1):1022.

23. Bhawana DR. Comparison of vibraject with conventional syringe during local anesthesia administration. IOSR J Dent Med Sci (IOSRJDMS) 2019;18(2):08-12. 